\title{
Rendimiento académico en función del estilo de aprender en estudiantes universitarios.
}

Academic performance based learning style in college students.

\section{Claudia Esperanza Saavedra Bautista ${ }^{1}$ Luis Alfonso Salcedo Plazas ${ }^{2}$}

Saavedra B. Claudia E, Salcedo P. Luis A. miradas Nº13 - 2015. ISSN: 0122 994X Págs 55 - 67

Recepción: Mayo 12 de 2015

Aprobación: Octubre 8 de 2015

Publicación: Diciembre 17 de 2015

\section{Resumen}

Este artículo es resultado de la implementación de guías didácticas diseñadas a partir de los estilos de aprendizaje de estudiantes universitarios como estrategia para mejorar su rendimiento académico. Se presentan los estilos de aprendizaje de mayor predominancia, así como la relación con su rendimiento académico, entendiendo éste como el nivel de conocimientos demostrados por los estudiantes en el marco del curso. Es un trabajo realizado bajo una modalidad presencial con cursos interdisciplinares entre los semestres I y VI de la facultad de ciencias de la educación de la Universidad Pedagógica y Tecnológica de Colombia. El estudio concluye que enseñar teniendo en cuenta el estilo de aprender de los estudiantes, puede mejorar su rendimiento académico y atender sus diferencias individuales, lo que propicia la construcción de un currículo integrador en el que se reconoce la diversidad de saberes en el aula.

Palabras Clave: Estilo de aprendizaje, rendimiento académico, guía didáctica, ambiente virtual de aprendizaje.

1 Magíster en Tecnología Informática, Esp. Informática para la docencia, Licenciado en informática educativa, Grupo de Investigación CETIN, Facultad Ciencias de la educación, Universidad Pedagógica y Tecnológica de Colombia, claudia. saavedra@uptc.edu.co, Colombia.

2 Especialista en Matemática y Estadística aplicada, Licenciado en Matemáticas y Física, Grupo de Investigación Gamma, Universidad Pedagógica y Tecnológica de Colombia, salcedop@email.com, Colombia. 


\section{Abstract}

This article is the result of the implementation of educational tutorials designed from the learning styles of university students as a strategy to improve their academic performance.

The most predominant learning styles are presented, and the relationship with their academic performance, which is taken as the level of knowledge shown by students in the course framework. It is a work made under a presential modality with interdisciplinary courses between semesters I and VI of the science faculty of education of the Pedagogical and Technological University of Colombia. The study concludes that teaching considering the learning styles of the students can improve their academic performance and meet their individual differences, which favors the construction of an inclusive curriculum in which the diversity of knowledge in the classroom is recognized.

Key Words: learning style, academic achievement, tutorial, virtual learning environment.

\section{Introducción}

Este trabajo da cuenta del desarrollo del proyecto "Ambiente virtual de aprendizaje a partir de estilos de aprendizaje en nativos digitales", que se llevó a cabo en el marco del grupo de investigación CETIN (Ciencia y Educación en Tecnología e informática), en la línea de investigación Innovaciones pedagógicas en tecnología e informática, de la Universidad Pedagógica y Tecnológica de Colombia. El proyecto se gesta con la intención de identificar la forma cómo aprenden los estudiantes que cursan la asignatura TIC y Ambientes de Aprendizaje de la Facultad de Ciencias de la Educación de la UPTC y sí enseñarles a través de su estilo de aprendizaje influye en su desempeño académico.

La relación estilo de aprendizaje estrategia de aprendizaje es un binomio que articulado debe permear en el desempeño académico del estudiante. Es así, que el presente estudio a través de la identificación de estilos de aprendizaje predominantes en los estudiantes formula guías didácticas que respondan a su forma de aprender. El trabajo reconoce la importancia de explorar los intereses y las diferencias individuales de los estudiantes que asisten a un aula de clase; ya que cuando se tiene en cuenta la forma cómo aprende el estudiante se rompe el paradigma de enseñanza para "estudiantes del común" en un ambiente regular, y se propone la enseñanza inclusiva, flexible, diversa e innovadora, donde el docente como actor importante del proceso de aprendizaje es capaz la provocar las potencialidades intelectuales de sus alumnos para contribuir con una transformación social pensada desde el conocimiento completo del estudiante.

\section{Perspectiva teórica Estilos de Aprendizaje}

Los estilos de aprendizaje se han vuelto una temática de interés en escenarios educativos que refleja la preocupación de los docentes por conocer la forma de aprender de sus estudiantes. Según Gallego (2008) "Las investigaciones cognitivas han demostrado que las personas piensan de manera distinta, captan la información, la procesan, la almacenan y la recuperan de forma diferente", por lo que resulta oportuno atender a la diversidad cognitiva de los estudiantes para ofrecer ambientes de aprendizaje acordes a sus formas de aprender. Los estilos de aprendizaje también se han definido como "rasgos cognitivos, afectivos y fisiológicos, que sirven como indicadores relativamente 
estables, de cómo los discentes perciben, interaccionan y responden a sus ambientes de aprendizaje" (Keefe, 1988). Así mismo, éste concepto se concibe como "la manera en la que un aprendiz comienza a concentrarse sobre una información nueva y difícil, la trata y la retiene"(Dunn \& Dunn, 1985).

Para adelantar la identificación de los estilos de aprendizaje en los estudiantes se utilizó el test CHAEA propuesto por Alonso, Gallego y Honey (1994). Este instrumento comprende 80 preguntas y se organiza en cuatro dimensiones cognitivas: activo, reflexivo, teórico y pragmático. Este test ha sido utilizado en diferentes escenarios académicos como instrumento diagnóstico para evaluar las dimensiones cognitivas de los sujetos. Los autores de este test realizaron su validación con una muestra de 1371 estudiantes universitarios de Madrid.

\section{Guías didácticas}

El estudio propone cuatro guías didácticas teniendo en cuenta la metodología de aprendizaje basado en problemas (ABP), donde la variación de la guía se dio en torno a los estilos de aprendizaje predominantes, estrategias y recursos web que podían responder de mejor forma al estilo de aprender de los estudiantes. Es decir, cada guía se organizó en 8 fases y para el desarrollo de cada una, se hizo una revisión y selección de herramientas web que favorecieran las dimensiones de personalidad de cada estilo de aprendizaje. La implementación de las guías didácticas se efectuó a través de una plataforma de aprendizaje en línea.

El aprendizaje basado en problemas (ABP), se sitúa dentro de la pedagogía activa y consiste en enfrentar a los estudiantes a situaciones problema que les genere conflicto cognitivo encaminado a una solución colectiva. Esta metodología de trabajo propicia momentos de reflexión compartida y persigue el desarrollo de habilidades donde prima el trabajo en equipo. Así mismo, en esta metodología la toma de decisiones resulta fundamental para lograr los objetivos planeados en el tiempo previsto. En el aprendizaje basado en problemas "el problema que se presenta en el escenario debe tener la suficientemente complejidad para que solamente se logre su solución trabajando cooperativamente" (Duch, 2001).

La diferencia entre el Aprendizaje basado en problemas y el aprendizaje clásico, radica en que en el $A B P$ parte de un problema que a medida que el estudiante se acerca a la solución va desarrollando su aprendizaje; mientras en el aprendizaje tradicional, se realiza un ejercicio lineal donde se expone lo que debe saber el estudiante como primera medida y luego se plantea el problema para que aplique lo aprendido. Según Wood (1993) "El mejor ABP evita enseñar a los estudiantes el contenido antes de que ellos se enfrenten al escenario. Los estudiantes deben orientarse para investigar la situación, generar las preguntas adecuadas, y producir su plan propio para resolver el problema".

Dentro de la revisión realizada se encontraron tres propuestas para aplicar el aprendizaje basado en problemas como son:

Torp y Sage (1998) en su libro "Aprendizaje basado en problemas", plantea que el ABP se lleva a cabo a partir de 8 etapas y que el aspecto más importante del diseño del ABP es el de centrar a los alumnos en un problema no estructurado. Las etapas sugeridas por estos autores son:

- Presentar el problema

- Identificar lo que sabemos, lo que nos hace falta saber y nuestras ideas

- Definir el enunciado problema 
- Reunir y compartir información

- Generar posibles soluciones

- Determinar el mejor haz de soluciones

- Presentar la solución

- Hacer un informe final sobre el problema

Otra propuesta de aprendizaje basado en problemas es la que plantea Exley y Dennick (2007) quienes señalan siete fases del proceso a tener en cuenta y son:

- Aclarar términos y conceptos

- Definir los problemas

- Analizar los problemas: preguntar, explicar, formular, hipótesis, etc.

- Hacer una lista sistemática del análisis

- Formular los resultados del aprendizaje esperados

- Aprendizaje independiente centrado en resultados

- Sintetizar y presentar nueva información

Morales y Landa (2004) establecen que el desarrollo del ABP ocurre en ocho fases organizadas de la siguiente manera:

- Leer y analizar el escenario del problema

- Realizar una lluvia de ideas

- Hacer una lista con aquello que se conoce

- Hacer una lista con aquello que no se conoce

- Hacer una lista de aquello que necesita hacerse para resolver el problema

- Definir el problema

- Obtener información

- Presentar resultados

Luego de realizar una revisión a los tres escenarios planteados anteriormente se seleccionó la propuesta de Morales y Landa (2004) atendiendo a los criterios que se ilustran en las siguientes tablas.

\begin{tabular}{|c|c|c|c|}
\hline \multicolumn{4}{|c|}{ APRENDIZAJE BASADO EN PROBLEMAS } \\
Torp y Sage (1998)
\end{tabular}

Tabla 1. Evaluación ABP propuesto por Torp y Sage (1998).

Fuente: Elaboración propia basada en la teoría de Torp y Sage (1998).

\begin{tabular}{|l|c|c|c|}
\hline \multicolumn{4}{|c|}{$\begin{array}{c}\text { APRENDIZAJE BASADO EN PROBLEMAS } \\
\text { Exley y Dennick (2007) }\end{array}$} \\
\hline \multicolumn{1}{|c|}{ FASES } & Análisis & Crítica & Cooperación \\
\hline $\begin{array}{l}\text { Aclarar términos y } \\
\text { conceptos }\end{array}$ & $\mathrm{X}$ & & \\
\hline Definir los problemas & $\mathrm{X}$ & & \\
\hline $\begin{array}{l}\text { Analizar los problemas: } \\
\text { preguntar, } \\
\text { formular, hipótesis, etc. }\end{array}$ & & $\mathrm{X}$ & $\mathrm{X}$ \\
\hline $\begin{array}{l}\text { Hacer una lista sistemática } \\
\text { del análisis }\end{array}$ & $\mathrm{X}$ & & \\
\hline $\begin{array}{l}\text { Formular los resultados del } \\
\text { aprendizaje esperados }\end{array}$ & $\mathrm{X}$ & & $\mathrm{X}$ \\
\hline $\begin{array}{l}\text { Aprendizaje independiente } \\
\text { centrado en resultados }\end{array}$ & & & \\
\hline $\begin{array}{l}\text { Sintetizar y presentar nueva } \\
\text { información }\end{array}$ & & $\mathbf{1}$ & $\mathbf{3}$ \\
\hline \multicolumn{1}{|c|}{ TOTAL } & $\mathbf{5}$ & & \\
\hline
\end{tabular}

Tabla 2. Evaluación ABP propuesto por Exley y Dennick (2007).

Fuente: Elaboración propia basada en la teoría de Exley y Dennick (2007). 


\begin{tabular}{|l|c|c|c|}
\hline \multicolumn{4}{|c|}{$\begin{array}{c}\text { APRENDIZAJE BASADO EN PROBLEMAS } \\
\text { Morales y Landa (2004) }\end{array}$} \\
\hline FASES & Análisis & Crítica & Cooperación \\
\hline $\begin{array}{l}\text { Leer y analizar el escenario } \\
\text { del problema }\end{array}$ & $\mathrm{X}$ & & \\
\hline Realizar una lluvia de ideas & $\mathrm{X}$ & & $\mathrm{X}$ \\
\hline $\begin{array}{l}\text { Hacer una lista con aquello } \\
\text { que se conoce }\end{array}$ & $\mathrm{X}$ & & $\mathrm{X}$ \\
\hline $\begin{array}{l}\text { Hacer una lista con aquello } \\
\text { que no se conoce }\end{array}$ & $\mathrm{X}$ & & $\mathrm{X}$ \\
\hline $\begin{array}{l}\text { Hacer una lista de aquello } \\
\text { que necesita hacerse para } \\
\text { resolver el problema }\end{array}$ & $\mathrm{X}$ & $\mathrm{X}$ & \\
\hline $\begin{array}{l}\text { Definir el problema } \\
\text { Obtener información }\end{array}$ & $\mathrm{X}$ & $\mathrm{X}$ & \\
\hline Presentar resultados & $\mathrm{X}$ & & $\mathrm{X}$ \\
\hline \multicolumn{1}{|c|}{ TOTAL } & $\mathbf{8}$ & $\mathbf{2}$ & $\mathbf{4}$ \\
\hline
\end{tabular}

Tabla 3. Evaluación ABP propuesto por Morales y Landa (2004).

Fuente: Elaboración propia basada en la teoría de Morales y Landa (2004).

Las ponderaciones obtenidas luego del proceso de evaluación de las metodologías, refieren utilizar como marco de referencia para el diseño de las guías didácticas el Aprendizaje Basado en Problemas propuesto por Morales y Landa (2004), ya que sus etapas promueven el análisis y la postura crítica de los estudiantes, así como algunas estrategias de cooperación que pueden incurrir en su mejoramiento académico. El concepto de rendimiento académico se trabajó bajo la perspectiva de Chadwick (1979) quién lo reconocer como "la expresión de capacidades y de características psicológicas del estudiante desarrolladas y actualizadas a través del proceso de enseñanza- aprendizaje que le posibilita tener un nivel de funcionamiento y logros académicos a lo largo de un período o semestre, que se sintetiza en un calificativo final (cuantitativo en el mayor de los casos) evaluador del nivel alcanzado" y desde la mirada de Nováez (1986), que afirma que: "el rendimiento académico es el quantum obtenido por el individuo en determinada actividad académica"

\section{Método}

Este proceso de investigación se desarrolló en el marco del grupo de investigación Ciencia y Educación en Tecnología e informática, (CETIN) bajo una metodología cuasi-experimental con enfoque cuantitativo, donde se estudiaron posibles relaciones de causa y efecto entre el diseño de guías didácticas partir del estilo de aprendizaje del estudiante frente a su rendimiento académico.

La población con la que se llevó a cabo el presente estudio corresponde a estudiantes de la Facultad de Ciencias de la Educación de la Universidad Pedagógica y Tecnológica de Colombia que cursaban la asignatura Tic y Ambientes de Aprendizaje. Esta asignatura corresponde al área interdisciplinar del currículo de la Facultad, por lo que los grupos se terminan conformándose por estudiantes de diferentes edades, carreras y semestres. La edad de los estudiantes oscila entre los $17 \mathrm{y}$ 30 años. Cabe anotar que con el fin de poder establecer el comportamiento de la variable estilos de aprendizaje en los estudiantes de la facultad se encuestó a 452 estudiantes aunque para el proceso de implementación de las guías didácticas solamente se trabajó con los grupos experimentales y el grupo control, notando tendencias similares en los resultados.

\begin{tabular}{|c|c|c|c|c|}
\hline \multirow{2}{*}{ Grupos } & \multirow{2}{*}{ Asignación } & \multicolumn{3}{|c|}{ Secuencia de registro } \\
\cline { 3 - 5 } & & Pretest & Tratamiento & Postest \\
\hline $\begin{array}{l}\text { Grupo A } \\
\text { - Grupo 10 }\end{array}$ & NA & O1 & $\mathrm{X}$ & $\mathrm{O} 2$ \\
\hline $\begin{array}{l}\text { Grupo B - } \\
\text { Grupo 25 }\end{array}$ & NA & O3 & $\mathrm{X}$ & $\mathrm{O} 4$ \\
\hline Grupo C- & & & - & \\
Grupo 6 & & & & \\
\hline
\end{tabular}

Tabla 3. Modelo de trabajo con los grupos en observación. 


\section{Resultados}

Los resultados estadísticos producto de la aplicación del test CHAEA a los 452 estudiantes se registran en la siguiente gráfica. La estructura de la gráfica presenta las cuatro dimensiones que propone el test: activo, reflexivo, teórico y pragmático. Para los estudiantes que no clasificaron en alguna de estas categorías se propuso la categoría "más de un estilo de aprendizaje", dado que se encontró que hay estudiantes que desarrollan más de un estilo de aprendizaje, pero se desconoce cómo clasificarlos en estas cuatro dimensiones que propone el test.

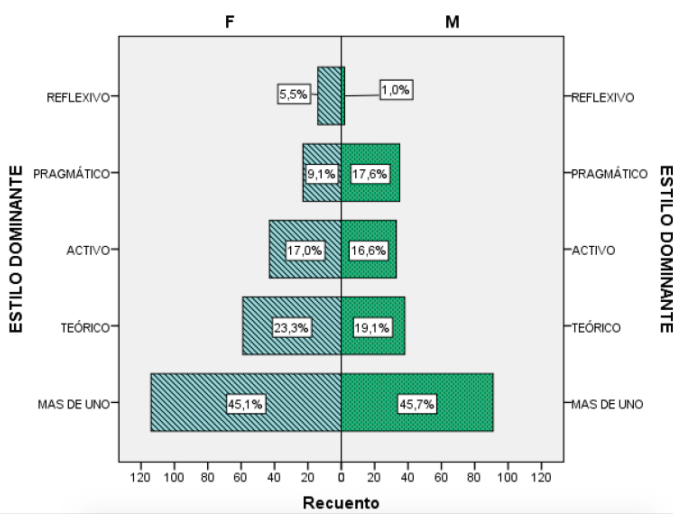

Gráfica 1. Resultados estilos de aprendizaje

Gráfica 1. Resultados estilos de aprendizaje

Fuente: Elaboración propia.

La anterior gráfica representa que de los 452 estudiantes encuestados el $5.5 \%$ de las mujeres y el $1 \%$ de los hombres tiene una predominancia al estilo de aprendizaje reflexivo; cifra que preocupa y que permite sugerir la necesidad de formular prácticas pedagógicas encaminadas a fortalecer la capacidad critica y reflexiva de los estudiantes. Del mismo modo con $17,6 \%$ los hombres demuestran tener un estilo de aprendizaje más pragmático en comparación con las mujeres quienes solo el 9,1\% tienen dominancia por este estilo de aprendizaje.
Para el caso del estilo de aprendizaje activo existe una proporción similar en los resultados de ambos géneros; es decir, en el $17 \%$ de las mujeres hay predominancia por el estilo de aprendizaje activo y en los hombres el $16,6 \%$. Por otro lado, el test arroja que el $23,3 \%$ de las mujeres es más teórico frente al 19,1\% que se manifiesta en los hombres.

Finalmente, existe una marcada proporción de personas que no se logra categorizar en las dimensiones que propone el test teniendo en cuenta que desarrollan más de un estilo de aprendizaje, pero se desconoce cómo clasificarlas en las cuatro categorías que propone el test.

Las siguientes gráficas ilustran los resultados obtenidos en los grupos experimentales A y B y el grupo control.

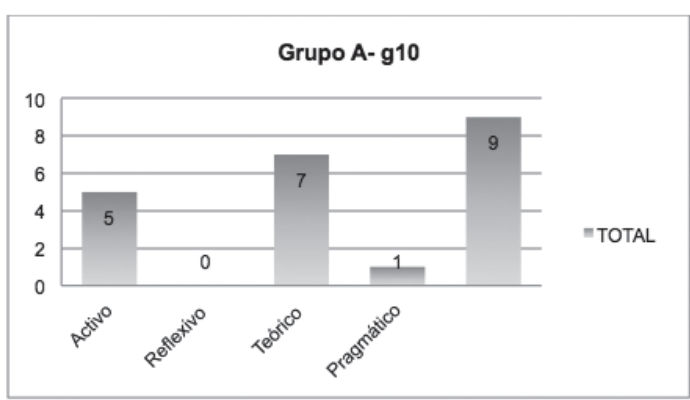

Gráfica 2. Resultados estilos de aprendizaje grupo experimental $A$. Fuente: Elaboración propia.

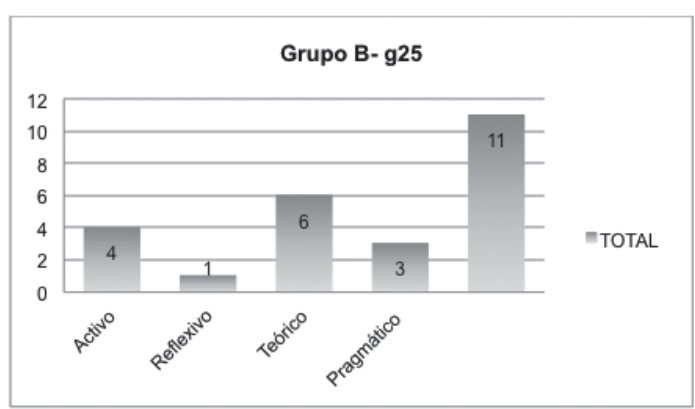

Gráfica 3. Resultados estilos de aprendizaje grupo experimental B. Fuente: Elaboración propia. 


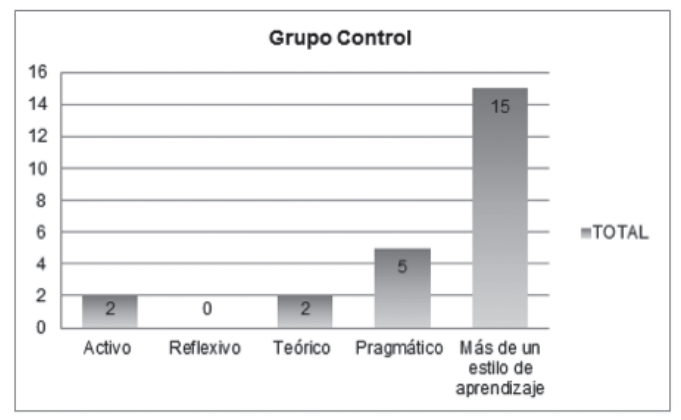

Gráfica 4. Resultados estilos de aprendizaje grupo control. Fuente: Elaboración propia.

Previo al estudio se recolectaron 452 registros para identificar los estilos de aprendizaje que predominaban de manera general en los estudiantes de la facultad y poder realizar comparaciones con los resultados de los grupos experimentales y el grupo control. Las anteriores gráficas permiten referir que existe una relación en las tendencias de los resultados entre estudiantes entrevistados previos al estudio (Gráfica 1.) y los grupos experimentales y el grupo control. (Gráfica 2, 3,4.)

\section{Diseño de las guías didácticas}

Una vez se determinaron los estilos predominantes en los estudiantes se procedió a diseñar cuatro guías didácticas teniendo en cuenta una temática de clase y en correspondencia con los estilos de aprendizaje que propone el test: activo, reflexivo, teórico y pragmático. Para el diseño de las guías se siguió la metodología ABP propuesta por Morales y landa (2004) y se realizó una búsqueda y evaluación de herramientas en línea que fortalecieran el estilo de aprendizaje de cada guía.

La estructura de las guías didácticas se pueden observar en la siguiente figura.

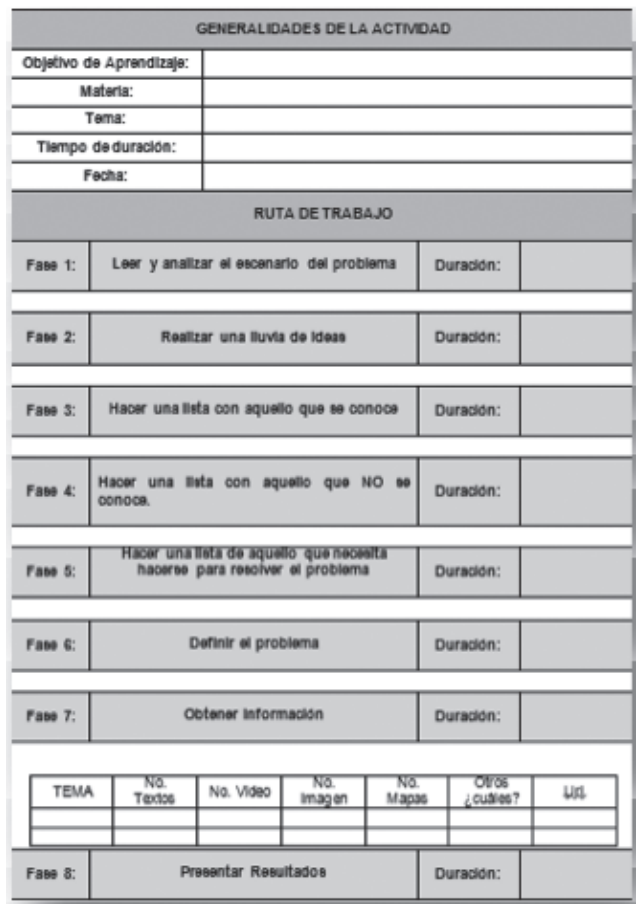

Figura 1. Estructura de las guías didácticas.

Fuente: Elaboración propia.

Teniendo en cuenta que la naturaleza de la asignatura en la que se desarrolló el estudio corresponde a TIC y Ambientes de Aprendizaje y que el objetivo de cada guía didáctica era fortalecer el estilo de aprendizaje de mayor predominancia de los estudiantes, se realizó una evaluación de herramientas web que favorecieran su estilo de aprender y facilitará el desarrollo de cada fase de la guía.

La evaluación de las herramientas se realizó teniendo en cuenta las dimensiones de personalidad propuestas por HoneyAlonso, (1994) para cada estilo de aprendizaje. 


\begin{tabular}{|c|c|c|c|c|c|c|}
\hline \multicolumn{7}{|c|}{ ESTILO DE APRENDIZAJE ACTIVO } \\
\hline \multirow{2}{*}{ 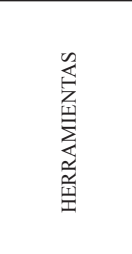 } & \multicolumn{5}{|c|}{$\begin{array}{l}\text { DIMENSIONES DE } \\
\text { PERSONALIDAD } \\
\text { Honey-Alonso, (1994) }\end{array}$} & \multirow{2}{*}{$\underset{\Leftrightarrow}{\stackrel{\leftrightarrow}{\leftrightarrows}}$} \\
\hline & 离 & 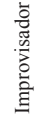 & 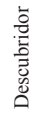 & 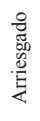 & 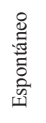 & \\
\hline $\begin{array}{l}\text { BLOGGER } \\
\text { (blog } \\
\text { Colaborativo) }\end{array}$ & $\mathrm{X}$ & $\mathrm{X}$ & $\mathrm{X}$ & $\mathrm{x}$ & $\mathrm{x}$ & 5 \\
\hline $\begin{array}{l}\text { PREZI } \\
\text { (Diseño de } \\
\text { presentaciones } \\
\text { dinámicas) }\end{array}$ & $\mathrm{x}$ & $\mathrm{X}$ & $\mathrm{X}$ & $\mathrm{x}$ & & 4 \\
\hline $\begin{array}{l}\text { GOOGLE } \\
\text { DRIVE } \\
\text { (Gestión de } \\
\text { archivos en la } \\
\text { nube) }\end{array}$ & & $X$ & $X$ & & & 2 \\
\hline $\begin{array}{l}\text { VOKI } \\
\text { (Diseño de } \\
\text { personajes } \\
\text { animados) }\end{array}$ & $X$ & $X$ & $x$ & & & 3 \\
\hline $\begin{array}{l}\text { MindMeister } \\
\text { (Diseño } \\
\text { de mapas } \\
\text { mentales) }\end{array}$ & & & & & & 0 \\
\hline $\begin{array}{l}z \\
0 \\
0 \\
0 \\
0 \\
z \\
0 \\
0\end{array}$ & \multicolumn{6}{|c|}{$\begin{array}{l}\text { Blogger sería la herramienta que permitirían fortalecer } \\
\text { el estilo de aprendizaje Activo en los Nativos digitales, } \\
\text { además de combinarse con otras herramientas } \\
\text { dinámicas como prezi y Voki }\end{array}$} \\
\hline
\end{tabular}

Tabla 4. Herramientas para el estilo de aprendizaje Activo.

\section{Descripción de las herramientas seleccionadas - Estilo Activo}

- Blogger: Esta herramienta estimula la creatividad de los usuarios y afianza procesos de toma de decisiones, promoviendo la construcción simultánea entre pares y la participación activa e interacción entre los mismos, de esta manera se ve favorecido el estilo de aprendizaje Activo, ya que se abren espacios para proponer ideas discutirlas, aplicarlas y explicarlas.

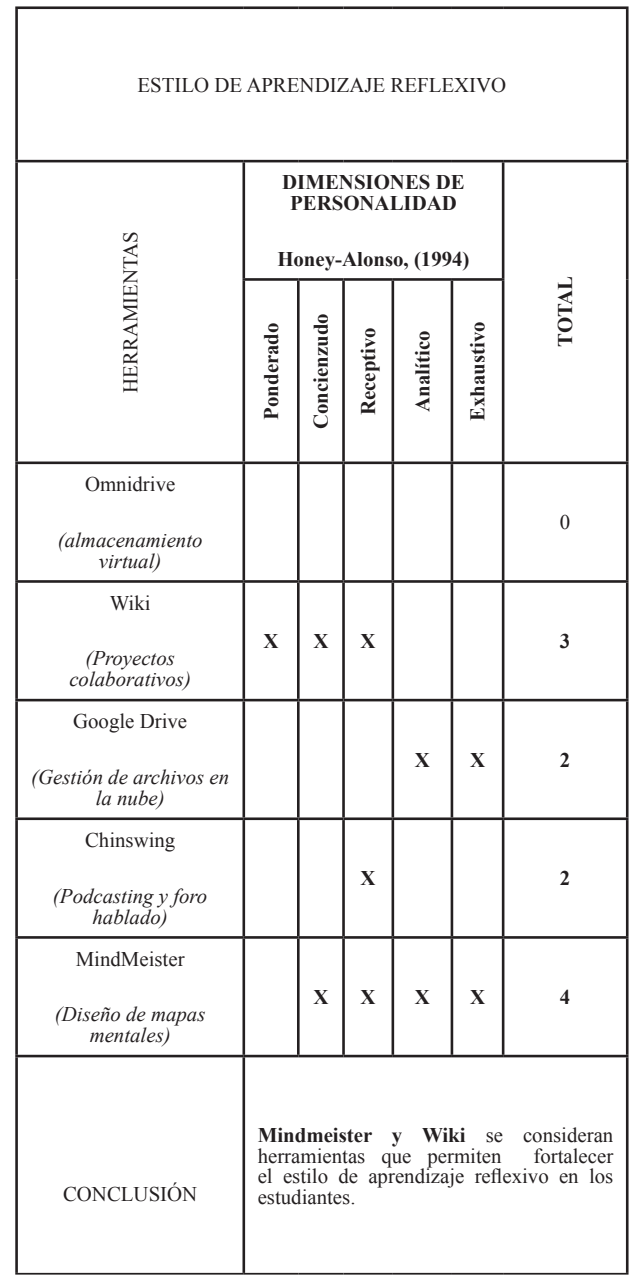

Tabla 5. Herramientas para el estilo de aprendizaje Reflexivo

\section{Descripción de las herramientas seleccionadas - Estilo Reflexivo}

- Mindmeister: Es una herramienta en línea que permite el diseño de mapas mentales en tiempo real; a través de esta herramienta los estudiantes logran construir y compartir conocimiento a partir de sus ideas promoviendo el enriquecimiento mutuo de los usuarios que participan en la construcción del mapa. Favorece el estilo de aprendizaje Reflexivo y Teórico en los estudiantes, 
y resulta pertinente teniendo en cuenta que dentro de los resultados preliminares que arrojaron los test aplicados se demuestra que existe un marcada debilidad en estos estilos de aprendizaje.

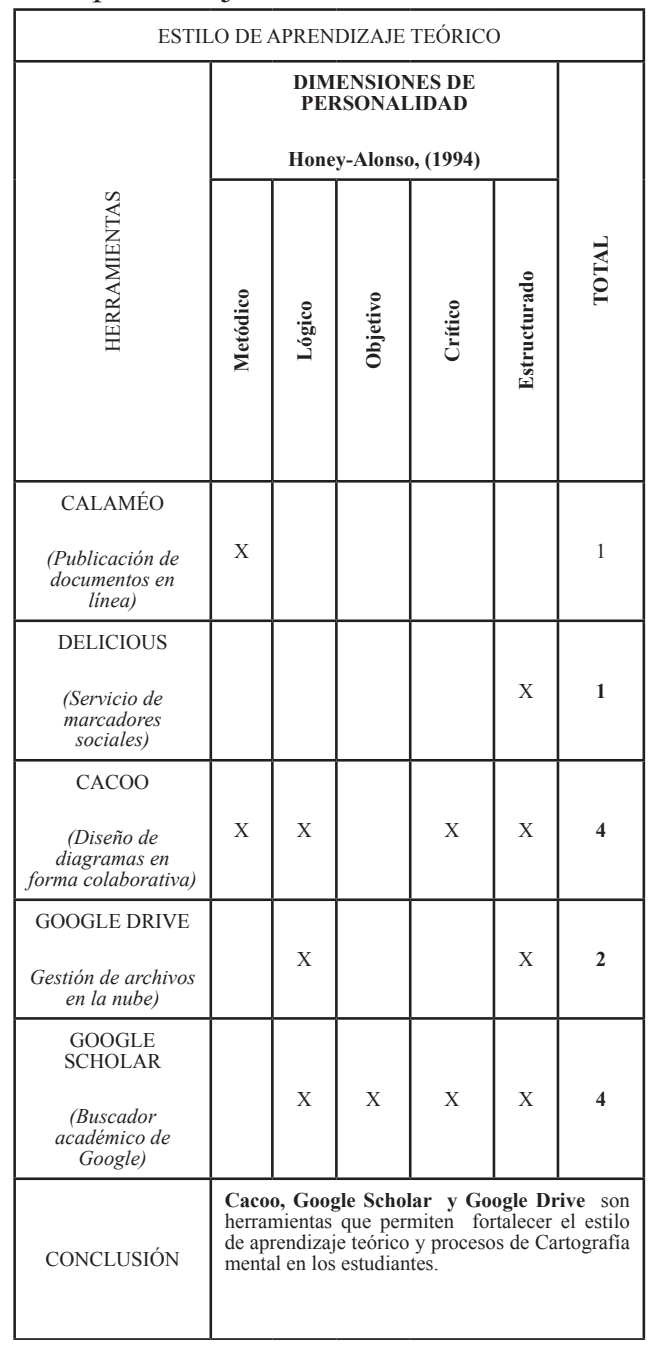

Tabla 6. Herramientas para el estilo de aprendizaje Teórico

\section{Descripción de las herramientas seleccionadas - Estilo Teórico}

- Cacoo: Herramienta gratuita de dibujo en línea con una interfaz gráfica amigable al usuario que permite crear una variedad de diagramas tales como mapas de sitio, wireframes, diagramas UML y de red. Permite al estudiante organizar sus ideas a través de diferentes esquemas gráficos.

- Google Scholar: Es el buscador académico de google que alberga un gran número de publicaciones científicas y actualizadas de diferentes áreas del saber. Favorece el estilo de aprendizaje teórico ya que provee al estudiante de diferentes fuentes de información de alta cientificidad como apoyo a su disciplina.

\begin{tabular}{|c|c|c|c|c|c|c|}
\hline \multicolumn{7}{|c|}{ ESTILO DE APRENDIZAJE PRAGMÁTICO } \\
\hline \multirow{3}{*}{ 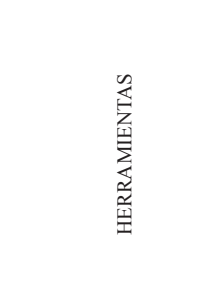 } & \multirow{2}{*}{\multicolumn{5}{|c|}{$\begin{array}{l}\text { DIMENSIONES DE } \\
\text { PERSONALIDAD } \\
\text { Honey-Alonso, (1994) }\end{array}$}} & \multirow{3}{*}{ 这 } \\
\hline & & & & & & \\
\hline & 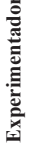 & : & 总 & 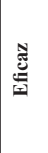 & 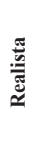 & \\
\hline $\begin{array}{c}\text { PREZI } \\
\text { (Diseño de } \\
\text { presentaciones } \\
\text { dinámicas) }\end{array}$ & $\mathbf{x}$ & $\mathbf{x}$ & & $\mathbf{x}$ & & 3 \\
\hline $\begin{array}{c}\text { Goanimate } \\
\text { (diseño de animaciones }\end{array}$ & $\mathbf{x}$ & $\mathbf{x}$ & $\mathbf{x}$ & $\mathbf{x}$ & & 4 \\
\hline $\begin{array}{l}\text { YOUTUBE } \\
\begin{array}{l}\text { (Subida de videos y } \\
\text { portal de videos) }\end{array}\end{array}$ & $\mathbf{x}$ & $\mathbf{X}$ & $\mathbf{x}$ & $\mathbf{x}$ & $\mathbf{X}$ & 5 \\
\hline $\begin{array}{c}\text { Wix } \\
\text { (Diseño de sitios web } \\
\text { dinámicos) }\end{array}$ & $\mathbf{x}$ & $\mathbf{x}$ & & $\mathbf{x}$ & & 3 \\
\hline $\begin{array}{c}\text { FLIXTIME } \\
\begin{array}{l}\text { (Diseño de videos en } \\
\text { línea) }\end{array}\end{array}$ & $\mathbf{x}$ & $\mathbf{X}$ & & & & 2 \\
\hline CONCLUSIÓN & $\begin{array}{l}\text { Yout } \\
\text { apren } \\
\text { pero } \\
\text { herra } \\
\text { pond }\end{array}$ & $\begin{array}{l}\text { ee } \\
\text { izaje } \\
\text { dicio } \\
\text { ienta } \\
\text { ación }\end{array}$ & $\begin{array}{l}\text { vede } \\
\text { Prag } \\
\text { al se } \\
\text { s qu } \\
\text { alta c }\end{array}$ & $\begin{array}{l}\text { forta } \\
\text { nático } \\
\text { pued } \\
\text { tan } \\
\text { omo: }\end{array}$ & $\begin{array}{l}\text { ecer } \\
\text { en } \\
\text { com } \\
\text { ién } \\
\text { anim }\end{array}$ & $\begin{array}{l}\text { tilo de } \\
\text { udiantes, } \\
\text { on otras } \\
\text { on una } \\
\text { Vix. }\end{array}$ \\
\hline
\end{tabular}

Tabla 7. Herramientas para el estilo de aprendizaje Pragmático 
Descripción de las herramientas seleccionadas - Estilo Pragmático

- Youtube: Es una herramienta que permite la búsqueda y subida en línea de videos, además permite compartirlos con los usuarios en la red. Favorece el estilo de aprendizaje Pragmático, ya que a través de los videos los estudiantes logran fortalecer destrezas de índole práctico y acceder al conocimiento de forma visual y práctica.

- Goanimate: Herramienta en línea que permite el diseño de videos y animaciones con múltiples personajes y objetos. Con GoAnime el estudiante puede desarrollar su capacidad creativa e innovadora para representar una situación presentada previamente favoreciendo así su intuición.

\section{Implementación de las guías didácticas}

La implementación de las guías didácticas se realizó a través de la plataforma edmodo. Esta herramienta combina el componente educativo con el social ofreciendo la posibilidad de trabajar de manera participativa e interactiva entre estudiantes y docentes. Esta aplicación se convierte en un espacio de aprendizaje dinámico donde se publica y evalúa contenido en línea. Además, tiene una versión móvil que permite estar conectado todo el tiempo para no perder las novedades de último momento, similar a una red social.

Teniendo en cuenta que las guías didácticas diseñadas estaban compuestas por ocho fases; en la medida que los estudiantes avanzaban en el desarrollo de las mismas, hacían sus entregas a través es este escenario de aprendizaje. Esta metodología permitió que los estudiantes trabajaran de manera colaborativa en torno a un problema planteado inicialmente y al que debían dar solución. La siguiente figura corresponde a la entrega del desarrollo de las fases de la guía didáctica en el escenario de aprendizaje.

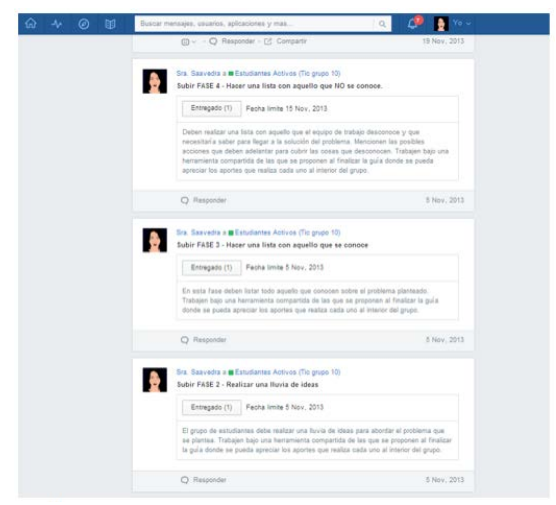

Figura 2. Entrega de resultados de cada fase Fuente: Captura de pantalla

\section{Análisis pre test $\mathrm{y}$ post test}

Para determinar si la implementación de las guías didácticas teniendo en cuenta el estilo de aprendizaje tuvo efectos positivos en el rendimiento académico de los estudiantes, se aplicó un pretest y post test a los grupos experimentales y al grupo control. Para estas pruebas se tuvo en cuenta la siguiente escala de valoración.

\begin{tabular}{|c|}
\hline ESCALADE VALORACIÓN \\
\hline 0 Deficiente \\
\hline $1-3$ Insuficiente \\
\hline $4-5$ Aceptable \\
\hline $6-8$ Sobresaliente \\
\hline 9 Excelente \\
\hline$X$ No presento \\
\hline
\end{tabular}

Tabla 8. Escala de evaluación del pre test y post test.

Fuente: Elaboración propia. 


\section{Resultados grupos experimentales}

A continuación se presenta el análisis de los resultados obtenidos luego de la aplicación del pre test y pos test en los grupos experimentales y en el grupo control.

\section{Resultados pre test $y$ post test grupos Experimentales A-B}

Al realizar la prueba de Homogeneidad Marginal Asintótica (Asymptotic Marginal-Homogeneity Test) empleando la función $\mathrm{mh}$ - test() del paquete estadístico $\mathrm{R}$, se obtiene un chi-cuadrado de 23.1974 con 4 grados de libertad y un valor-p de 0.0001156 . Es decir que existe suficiente evidencia para afirmar que no hay homogeneidad marginal en la tabla $5 \times 5$ construida con las diferentes categorías de clasificación empleadas en el Pre test y en el Post test; lo cual equivale a afirmar que el grupo de estudiantes, que participaron en el estudio, sufrieron cambios en su desempeño durante el período que duró la investigación; a manera de ejemplo, estudiantes que inicialmente se clasificaron como "aceptable" en el Pre test, se clasifican como "sobresaliente" en el Post test. En la siguiente gráfica se ilustra el resultado de los grupos A-B (Experimentales) en el Pre y Post test.

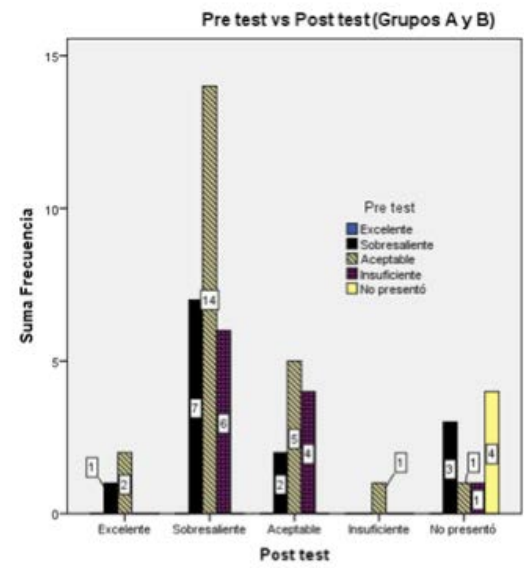

Gráfica 5. Resultados pre test y post test grupo Experimental $A-B$.Fuente: Elaboración propia.

\section{Resultados grupo control}

Al realizar la prueba de Homogeneidad Marginal Asintótica (Asymptotic MarginalHomogeneity Test), empleando la función mh test() del paquete estadístico $\mathrm{R}$, se obtiene un chi-cuadrado de 3.8525 con 3 grados de libertad y un valor-p de 0.2778 . Es decir que no existe suficiente evidencia para afirmar que hay homogeneidad marginal en la tabla $4 \times 4$ (se omite la categoría "excelente" puesto que todas las entradas en la tabla tienen un valor de cero) construida con las diferentes categorías de clasificación empleadas en el Pre test y en el Post test; lo cual equivale a afirmar que el grupo de estudiantes, que participaron en el estudio, no sufrieron cambios significativos en su desempeño durante el período que duró la investigación; a manera de ejemplo, el grupo de estudiantes que inicialmente se clasificaron como "aceptable" en el Pre test, se clasifican nuevamente en esta categoría en el Post test. En la siguiente gráfica se ilustran los resultados.

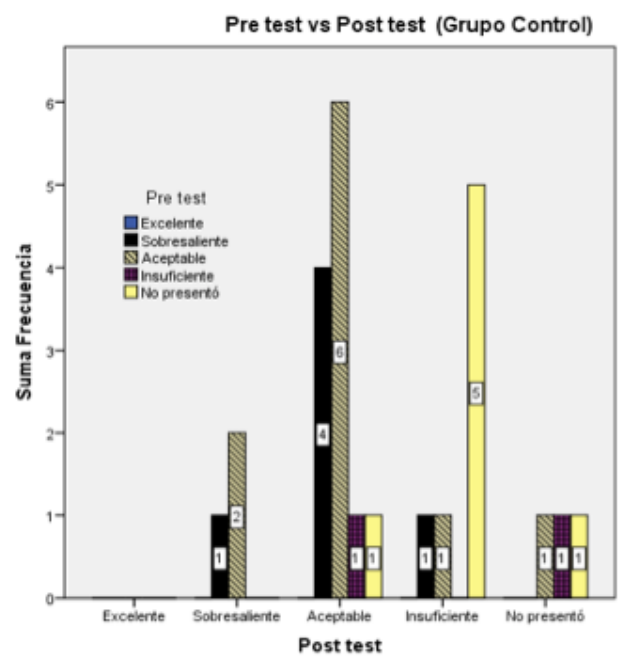

Gráfica 8. Resultados pre test VS. Post test grupo control. Fuente: Elaboración propia. 


\section{Discusión}

El estudio adelantado permite demostrar que en la medida que se enseña al estudiante teniendo en cuenta su estilo de aprendizaje, el rendimiento académico tiende a mejorar, ya que estudiantes que inicialmente se clasificaron como "aceptable" en el Pre test, se clasifican como "sobresaliente" en el Post test. Hallazgos similares son los que se reportan en el estudio realizado en la universidad tecnológica de Bolívar por J. Ruíz, and J. Morales (2006), denominado "Estilos de aprendizaje y rendimiento académico en estudiantes universitarios", el cuál explora la existencia de alguna relación entre los estilos de aprendizaje $\mathrm{y}$ el rendimiento académico de los estudiantes universitarios, arrojando como resultado una preferencia hacia el estilo de aprendizaje, activo, teórico y pragmático y un bajo porcentaje en la predominancia del estilo de aprendizaje reflexivo. Así mismo, encontraron que existe una correlación positiva entre el estilo de aprendizaje teórico y el rendimiento académico de los estudiantes, resultado que es similar con el obtenido en el presente estudio.

Otro trabajo de similar intención es el desarrollado por D. Gallego y M. García, (2012), titulado "Los estilos de aprendizaje en la formación inicial de docentes", el cual impulsa la calidad de formación inicial del docente y señala la importancia de considerar que una acción concreta comienza por hacer realidad la implementación de la diversidad de estilos de aprendizaje. El estudio concluye que las metodologías líderes del siglo XXI deben desarrollarse desde el conocimiento completo del estudiante, de lo contrario se corre el riesgo de caer en una actividad estéril.

\section{Conclusión}

Los resultados estadísticos permiten referir que efectivamente en la medida que se adopten estrategias de enseñanza en función del estilo de aprender del estudiante, se puede mejorar el nivel de desempeño y alcance de los objetivos de aprendizaje. (Gráfica 5).

Resulta oportuno conocer cómo categorizar a los estudiantes que no clasifican en las dimensiones que proponen los test; es decir, a los estudiantes que tienen más de un estilo de aprendizaje dominante, debido a que el $45,1 \%$ de los estudiantes de la facultad de educación de la UPTC pueden ser definidos como estudiantes multitareas, tal como lo refiere la literatura y en consecuencia pueden desarrollar más de un estilo de aprendizaje, permitiendo que un mayor número de actividades propuestas por el docente intervengan en su aprendizaje.

Identificar los estilos de aprendizaje de los estudiantes en el aula, es una labor que permite la construcción de un currículo integrador que reconozca la diversidad como factor clave en la enseñanza, evitando la planeación de prácticas pedagógicas para estudiantes estándar, pensadas desde el interés del docente, sino por el contrario formuladas desde el conocimiento pleno del estudiante.

Someter a los estudiantes a recetas de clases universales desconociendo ingredientes como el estilo de aprender, dificulta su óptimo desempeño académico y desaprovecha el potencial que sus dimensiones de personalidad pueden aportar en su proceso de aprendizaje. (Gráfica 8). 


\section{Agradecimientos}

Los autores expresan su agradecimiento a la Universidad Pedagógica y Tecnológica de Colombia por el espacio otorgado para realizar la investigación.

\section{Referencia Bibliográfica}

Alonso, C.; Gallego, D. and Honey, P. (1994). "Los estilos de aprendizaje: procedimientos de diagnóstico y mejora", Ediciones Mensajero, Bilbao.

Alonso, C.; Gallego, D. and Honey, P. (1999). Los estilos de aprendizaje $5^{\text {a }}$ ed. Bilbao: Mensajero.

Axelrod, R., (1981).The Evolution of Cooperation. NY, United States: Basic Books, 1981.

Batista, M. Á. H. (2006). Consideraciones para el diseño didáctico de ambientes virtuales de aprendizaje: una propuesta basada en las funciones cognitivas del aprendizaje. Revista Iberoamericana de Educación, 38(5), 2.

Bueno, P. M., \& Fitzgerald, V. L. (2004). Aprendizaje Basado En Problemas ProblemBased Learning. Theoria, 13, 145-157.

Camarero Suárez, F. J., Martín del Buey, F. D. A., \& Herrero Díez, F. J. (2000). Estilos y estrategias de aprendizaje en estudiantes universitarios. Psicothema, 12 (4).

Chadwick (1979) citado en Vallejo, A. P., \& Risoto, M. A. (2014). La variable género y su relación con el autoconcepto y el rendimiento académico de alumnado universitario. Etic@ net, 1(14), 124-140.

Duch, Barbara. (2001). Problems: A Key Factor in PBL. Center for Teaching Effectiveness University of Delaware.

Dunn, R. S., Dunn, K. J., \& Price, G. E. (1989). Learning style inventory (LSI). Price Systems, Incorporated (PO Box 1818, Lawrence 66044).

Exley, K. y Dennis, R. Enseñanza en pequeños grupos en Educación Superior. Madrid: Narcea, 2007.
Gallego, D. (2012). Los estilos de aprendizaje en la formación inicial del docente. Learning styles-in initial teacher. Revista Estilos de Aprendizaje, no. 9, Vol 9.

Gallego, D. (2008). Los estilos de aprendizaje y la enseñanza de las matemáticas. Revista Complutense De Educación, 19(1),2008,

Gallego, D. and Alonso, C., (2008). "Estilos de Aprender en el siglo XXI".

García, C. M. A., Honey, P., \& Gil, D. J. G. (1994). Los estilos de aprendizaje: procedimientos de diagnóstico y mejora.

Keefe, J. (1988). Aprendiendo Perfiles de Aprendizaje. Asociación Nacional de Escuelas Secundrias.

Morales, P. y Landa, V. (2004). Aprendizaje basado en problemas, en Theoria, Vol.13. Págs. 145-157.

Nováez, M. (1986). Psicología de la actividad escolar. México: Editorial Iberoamericana.

Ruiz, B. L. R., Gamboa, J. T., \& Arrieta, J. M. (2006). Estilos de aprendizaje y rendimiento académico en estudiantes universitarios. Revista galego-portuguesa de psicoloxía e educación: revista de estudios e investigación en psicología y educación, (13), 441-460.

Torp, L., \& Sage, S. (1998). El aprendizaje basado en problemas: desde el jardín de infantes hasta el final de la escuela secundaria. Amorrortu.

Wood (1993) citado en Romero-Álvarez, J. G., Rodríguez-Castillo, A., \& GómezPérez, J. (2008). Evaluación de escenarios para el aprendizaje basado en problemas (ABP) en la asignatura de química de bachillerato. Educación química, 19(3), 195200. 